\title{
Condensed Nearest Neighbor Data Domain Description
}

\author{
Fabrizio Angiulli
}

\begin{abstract}
A simple yet effective unsupervised classification rule to discriminate between normal and abnormal data is based on accepting test objects whose nearest neighbors' distances in a reference data set, assumed to model normal behavior, lie within a certain threshold. This work investigates the effect of using a subset of the original data set as the reference set of the classifier. With this aim, the concept of a reference-consistent subset is introduced and it is shown that finding the minimum-cardinality referenceconsistent subset is intractable. Then, the Condensed Nearest Neighbor Domain Description (CNNDD) algorithm is described, which computes a reference-consistent subset with only two reference set passes. Experimental results revealed the advantages of condensing the data set and confirmed the effectiveness of the proposed approach. A thorough comparison with related methods was accomplished, pointing out the strengths and weaknesses of one-class nearest-neighbor-based training-set-consistent condensation.
\end{abstract}

Index Terms-Classification, data domain description, data condensation, nearest neighbor rule, novelty detection.

\section{INTRODUCTION}

$\mathrm{D}$ ATA domain description, also called one-class classification, is a classification technique whose goal is to distinguish between objects belonging to a certain class and all the other objects of the space. The task that needs solving in one-class classification is the following: Given a data set of objects, called training or reference set, belonging to a certain object space, find a description of the data, that is, a rule partitioning the object space into an accepting region, containing the objects belonging to the class represented by the training set, and a rejecting region, containing all the other objects. Data domain description is related to outlier or novelty detection, as the description of the data is then used to detect the objects deviating significantly from the training data.

Given a data set, also called reference set, of objects from an object space and two parameters $k$ and $\theta$, we call the Nearest Neighbor Domain Description (NNDD) rule the classifier that associates a feature vector $\delta(p) \in \mathbb{R}^{k}$ with each object $p$. The elements of $\delta(p)$ are the distances of $p$ to its first $k$-nearest neighbors in the reference set and the classifier accepts $p$ iff $\delta(p)$ belongs to the hypersphere (according to one of the $L_{r}$ Minkowski metrics $\left.r \in\{1,2, \ldots, \infty\}\right)$ centered at the origin of $\mathbb{R}^{k}$ and having radius $\theta$, that is, iff $\|\delta(p)\|_{r} \leq \theta$.

The contribution of this work can be summarized as follows: The concept of reference-consistent subset for the NNDD rule, which is a subset of the reference set that correctly classifies all the objects in the reference set, is defined, and the relationship between the generalization of the NNDD classifier and the size of the reference set is pointed

- The author is with the Dipartimento di Elettronica Informatica $e$ Sistemistica, Università della Calabria, Via P. Bucci, 41C 87036 Rende (CS), Italy. E-mail: f.angiulli@deis.unical.it.

Manuscript received 28 Mar. 2006; revised 8 Oct. 2006; accepted 27 Nov. 2006; published online 18 Jan. 2007.

Recommended for acceptance by J. Hu.

For information on obtaining reprints of this article, please send e-mail to: tpami@computer.org, and reference IEEECS Log Number TPAMI-0246-0306. Digital Object Identifier no. 10.1109/TPAMI.2007.1086.

0162-8828/07/\$25.00 (C) 2007 IEEE out, concluding that replacing the original reference set with a reference-consistent subset improves space requirements, response time, and generalization. It is shown that finding the minimum-cardinality reference-consistent subset is a computationally demanding task and the Condensed Nearest Neighbor Domain Description (CNNDD) algorithm is provided, which computes a reference-consistent subset with only two data set passes. Experimental results show that the CNNDD algorithm achieves notable training set reduction and maintains, or even improves, the accuracy of the NNDD rule. Then, the CNNDD algorithm is compared with related approaches, pointing out the strengths and weaknesses of one-class nearest-neighbor-based training-set-consistent condensation. To conclude, robustness to noise and outliers is investigated.

The rest of the paper is organized as follows: In Section 2, the relationship between the approach here proposed and relevant literature is presented. In Section 3, the NNDD rule and the concept of the reference-consistent subset are formally defined. In Section 4, the CNNDD algorithm is described. Section 5 reports experimental results and comparison with related methods. Finally, in Section 6, conclusions are drawn.

\section{Related Work}

The literature related to this work can be grouped into three main categories: nonparametric binary classification using the nearest neighbor rule, one-class classification, and outlier detection. Next, these approaches are briefly described.

In the nonparametric binary classification problem, there is an available traininqg set $\left\{\left(x_{1}, y_{1}\right), \ldots,\left(x_{n}, y_{n}\right)\right\}$ of $n$ pairs $\left(x_{i}, y_{i}\right), 1 \leq i \leq n$, where $x_{i}$ is an object from an object space, and $y_{i} \in\{-1,1\}$ is the corresponding class label. The nearest neighbor rule (1-NN rule) [12] assigns the label $y_{j}$ to a new object $q$, where $x_{j}$ is the nearest neighbor of $q$ in $\left\{x_{1}, \ldots, x_{n}\right\}$ according to a certain metric. This rule is based on the property that the nearest neighbor $x_{j}$ of $q$ contains at least half of the total discrimination information contained in an infinite-sized training set [6], [23], [8]. The generalization of 
the 1-NN rule, which is the $k$-NN rule, in which a new pattern $q$ is classified into the class with the most members present among its $k$-nearest neighbors in $\left\{x_{1}, \ldots, x_{n}\right\}$, has the property that its probability of error asymptotically approaches the Bayes error [9]. Since the $k$-NN rule requires all the previously classified data to be stored, several techniques to reduce the size of the stored data have been proposed. Among these techniques, training-set-consistent ones aim at selecting a subset of the training set that classifies the remaining data correctly [15], [7], [4], [1]. These methods have the same goal as the CNNDD, but the subset they extract is consistent for the $k$-NN-classification rule rather than for the NNDD rule. Thus, it is worth pointing out that, since the two decision rules are greatly different, the consistent subsets of the two rules are of a different nature. Even by disregarding the fact that the $k$-NN rule requires labeled data, whereas the NNDD works on unlabeled data, there is no way of obtaining a subset consistent for the latter rule starting from a subset consistent for the former or vice versa. Indeed, consider that objects belonging to one type of subset do not usually belong to the other type. This is because the condensed $k$-NN rule searches for the objects mostly contributing to form the boundary between the classes in the data set, whereas the CNNDD rule searches for objects distributed along the data set shape and central with respect to the overall population.

The one-class classification task has been previously introduced. There are several approaches to one-class classification. In the nearest neighbor one-class classification method NN-d [25], a test object $p$ is accepted if the distance to its nearest neighbor $q$ in the training set is less than or equal to the distance from $q$ to its nearest neighbor in the training set. This measure is comparable with the Local Outlier Factor [3] used to detect outliers. The $k$-center method covers the data set with $k$ balls with equal radii [28]. Ball centers are placed on training objects such that the maximum distance of all minimum distances between training objects and the centers is minimized. Placing balls is similar to solving the $k$-center problem introduced in the discrete location theory, where, given a set of cities, one has to pick $k$ cities and build warehouses in them so as to minimize the maximum distance of any city from its closest warehouse (see [16] for an example). One-class classification techniques based on Support Vector (SV) Machines (SVMs) extend the SV algorithm to the case of unlabeled data [21], [24] (see Section 5.2 for a better description of this technique). In order to properly model outlier or noise possibly in the training set, model-based approaches such as the one presented in [20] assume that the data set consists of a mixture of two populations: A regular population with distribution $\mu_{R E G}$ and an outlier population with distribution $\mu_{O U T}$. Assuming that these distributions are members of given parametric families, the goal is that of estimating their parameters. There are many other interesting approaches to one-class classification. The reader is referred to [26] for a comprehensive treatment.

Research on outlier detection in data mining focuses on providing techniques for identifying the most deviating objects in an input data set. Distance-based outlier detection was introduced in [17]: A point in a data set is a $D B(c, d)$ outlier with respect to parameters $c$ and $d$ if at least fraction $c$ of the points in the data set lies greater than distance $d$ from it. This definition generalizes several discordancy tests to detect an outlier given in statistics and it is suitable when the data set does not fit any standard distribution. The definition in [19] is closely related to the previous one: Given $k$ and $n$, a point $p$ is an outlier if not more than $n-1$ other points in the data set have a higher value of $D^{k}$ than $p$, where $D^{k}(p)$ denotes the distance of the $k$ th nearest neighbor of a point $p$. In order to take into account the sparseness of the neighborhood of a point, [2] considers the measure $w_{k}(p)$ for each point $p$, denoting the sum of the distances to its $k$-nearest neighbors. Eskin et al. [11] provide further algorithms for distance-based anomaly detection. We point out that the measure $\|\delta(p)\|_{r}$ used here generalizes all distance-based measures, since $D^{k}(p)=\|\delta(p)\|_{\infty}$, and $w_{k}(p)=\|\delta(p)\|_{1}$.

\section{THE NNDD RULE}

In the following, a set of objects is denoted with $U$ with d being a distance on $U, D$ a set of objects from $U, k$ a positive integer number, $\theta$ a positive real number, and $r$ a Minkowski metric $L_{r}$, that is, $r \in\{1,2, \ldots, \infty\}$.

Given an object $p$ of $U$, the $k$ th nearest neighbor $n n_{D, \mathrm{~d}, k}(p)$ of $p$ in $D$, according to d, is the object $q$ of $D$ such that there exist exactly $k-1$ objects $s$ of $D$ with $\mathrm{d}(p, s) \leq \mathrm{d}(p, q)$. If $p \in D$, then $n n_{D, \mathrm{~d}, 1}(p)=p$. The $k$-nearest neighbors' distances vector $\delta_{D, \mathrm{~d}, k}(p)$ of $p$ in $D$ is ${ }^{1}$

$$
\delta_{D, \mathrm{~d}, k}(p)=\left(\mathrm{d}\left(p, n n_{D, \mathrm{~d}, 1}(p)\right), \ldots, \mathrm{d}\left(p, n n_{D, \mathrm{~d}, k}(p)\right)\right) .
$$

The NNDD rule $\operatorname{NNDD}_{D, \mathrm{~d}, k, \theta, r}$ according to $D, \mathrm{~d}, k, \theta$, and $r$ is the function from $U$ to $\{-1,1\}$ such that

$$
\operatorname{NNDD}_{D, \mathrm{~d}, k, \theta, r}(p)=\operatorname{sign}\left(\theta-\left\|\delta_{D, \mathrm{~d}, k}(p)\right\|_{r}\right),
$$

where $\operatorname{sign}(x)=-1$ if $x<0$, and $\operatorname{sign}(x)=1$ otherwise.

Intuitively, the NNDD rule returns 1 when the object belongs to the class represented by $D$, whereas it returns -1 when the object does not belong to that class. In the special cases $k=1$ and $\theta=0$, the rule accepts an object $p$ iff $p \in D$, whereas for $k=1$ and $\theta>0$, the rule accepts an object if it lies in the neighborhood of radius $\theta$ of some object in $D$.

Let $f$ be $\operatorname{NNDD}_{D, \mathrm{~d}, k, \theta, r}$. The accepting region $\mathcal{R}(f)$ of $f$ is the set $\{x \in U \mid f(x)=1\}$. The rejecting region $\overline{\mathcal{R}}(f)$ of $f$ is the set $U \backslash \mathcal{R}(f)$. An object $x \in \overline{\mathcal{R}}(f)$ is said to be an outlier. The empirical risk or training set error of the NNDD classifier $f$ is the quantity

$$
R^{e m p}(f)=\frac{|D \cap \overline{\mathcal{R}}(f)|}{|D|} .
$$

The empirical risk is directly proportional to the value of $k$ and inversely proportional to the value of $\theta$. Indeed, $\left\|\delta_{D, \mathrm{~d}, k-1}(p)\right\|_{r} \leq\left\|\delta_{D, \mathrm{~d}, k}(p)\right\|_{r}$ for $k>1 . R^{e m p}(f)$ is certainly 0 for $k=1$ or for arbitrarily large values of $\theta$.

When the reference set $D$ is large, space requirements to store $D$ and time requirements to find the nearest neighbors of an object in $D$ increase. In the spirit of the reference set thinning problem for the $k$-NN rule [15], [27], the concept of NNDD reference-consistent subset is defined and then, it is shown that finding a minimum NNDD reference-consistent subset is NP-hard.

An NNDD reference-consistent subset of $D$ with respect to $\mathrm{d}, k, \theta$, and $r$ is a subset $S$ of $D$ such that

$$
(\forall p \in D)\left(\mathrm{NNDD}_{D, \mathrm{~d}, k, \theta, r}(p)=\operatorname{NNDD}_{S, \mathrm{~d}, k, \theta, r}(p)\right),
$$

that is, a subset of $D$ that correctly classifies the objects in $D$.

1. If there are less than $k$ objects in $D$, then (the last) $k-|D|$ elements of $\delta_{D, \mathrm{~d}, k}(p)$ are equal to $+\infty$. 
The complexity of finding a minimum reference-consistent subset is related to the complexity of the following decision problem: given an integer $m, 1 \leq m \leq|D|$, the NNDD minimum reference-consistent subset problem $\langle D, \mathrm{~d}, k, \theta, r, m\rangle$ is: Is there an NNDD reference-consistent subset $S$ of $D$ with respect to d, $k, \theta$, and $r$ such that $|S| \leq m$ ?

Theorem 1. Let $r \in \mathbb{N}^{+}$denote a finite Minkowski metrics $L_{r}$.

Then, the $\langle D, \mathrm{~d}, k, \theta, r, m\rangle$ problem is NP-complete.

Proof. Membership. Given a subset $S$ of $D$, having size $|S| \leq m$, it can be checked in polynomial time that for each $p \in D, \operatorname{NNDD}_{D, \mathrm{~d}, k, \theta, r}(p)=\operatorname{NNDD}_{S, \mathrm{~d}, k, \theta, r}(p)$.

Hardness. The proof is by reduction to the Dominating Set Problem [14]. Let $G=(V, E)$ be an undirected graph, and let $m \leq|V|$ be a positive integer. The Dominating Set Problem is: Is there a subset $U \subseteq V$, called dominating set of $G$, with $|U| \leq m$, such that for all $v \in(V-U)$ there exists $u \in U$ with $\{u, v\} \in E$ ?

Let $G=(V, E)$ be an undirected graph. Define the metric $\mathrm{d}_{V}$ on the set $V$ of the nodes of $G$ as follows: $\mathrm{d}_{V}(u, v)=1$ if $\{u, v\} \in E$ and $\mathrm{d}_{V}(u, v)=2$ otherwise. Let $\theta_{k, r}$ be $\left(1+2^{r}(k-1)\right)^{1 / r}$. Now, we prove that $G$ has a dominating set of size $m$ iff $\left\langle V, \mathrm{~d}_{V}, k, \theta_{k, r}, r, m\right\rangle$ is a "yes" instance.

First, we note that, for each $v \in V$,

$$
\left\|\delta_{V, \mathrm{~d}_{V}, k}(v)\right\|_{r} \leq\left(0+2^{r}(k-1)\right)^{1 / r} \leq \theta_{k, r} .
$$

$(\Rightarrow)$ Suppose that $G$ has a dominating set $U$ such that $|U| \leq m$. Then, $U$ is a reference-consistent subset of $V$ with respect to $\mathrm{d}_{V}, k, \theta_{k, r}, r$. Indeed, let $v$ be a generic object of $V$. If $v \in U$, then

$$
\left\|\delta_{U, \mathrm{~d}_{V}, k}(v)\right\|_{r} \leq\left(0+2^{r}(k-1)\right)^{1 / r}<\theta_{k, r} ;
$$

otherwise, $v \notin U$ and

$$
\left\|\delta_{U, \mathrm{~d}_{V}, k}(v)\right\|_{r} \leq\left(1+2^{r}(k-1)\right)^{1 / r} \leq \theta_{k, r} .
$$

$(\Leftarrow)$ Suppose that there is a reference-consistent subset $U$ of $V$ such that $|U| \leq m$. By contradiction, assume that there is $v \in(V-U)$ such that for each $u \in U$, $\{v, u\} \notin E$. Then, $\left\|\delta_{U, \mathrm{~d}_{V}, k}(v)\right\|_{r} \geq 2 k^{1 / r}>\theta_{k, r}$ and $U$ is not a reference-consistent subset of $V$. It follows immediately that $U$ is a dominating set for $G$.

Theorem 1 also holds for the special cases $k=1$ and $r=\infty$. It follows immediately from Theorem 1 that the problem of computing the minimum size reference-consistent subset is NP-hard.

Before concluding the section, the concept of sample compression scheme is recalled. A sample compression scheme is defined by a fixed rule $\sigma: D \mapsto \sigma(D)$ for constructing a classifier from a given set of data. Given a training set $D$, it is compressed by finding the smallest subset (the compression set) $S \subseteq D$ for which the classifier $\sigma(S)$ correctly classifies the whole set $D$. It is known that the size of a sample compression scheme can be used to bound generalization [18], [13].

It can be finally concluded from the discussion above that replacing the reference set $D$ with a reference-consistent subset $S$ of $D$ has a twofold usefulness: Both response time and generalization of the classifier are improved.

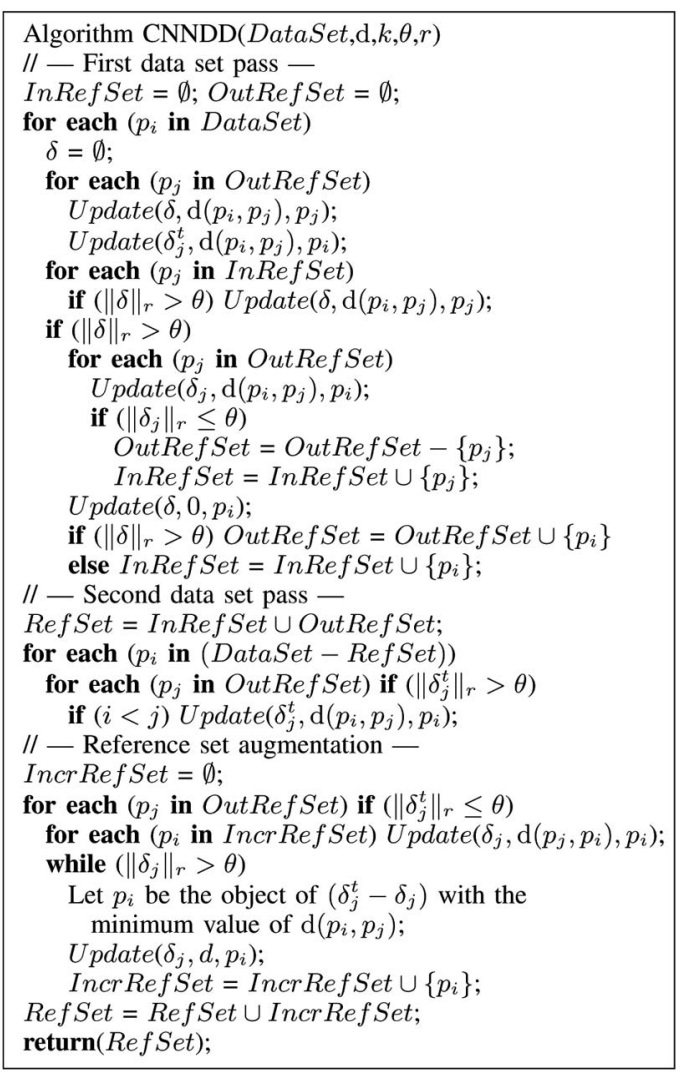

Fig. 1. The CNNDD rule.

\section{The Condensed NNDD Rule}

In this section, the CNNDD algorithm is described. This algorithm computes a reference-consistent subset for the NNDD rule with only two data set passes.

The algorithm, shown in Fig. 1, receives in input the data set DataSet and parameters d, $k, \theta$, and $r$ and outputs a reference-consistent subset RefSet. Let $f$ denote the classifier $\mathrm{NNDD}_{\text {DataSet, }, k, \theta, r}$. We recall that RefSet must be such that for each $p$ of DataSet, the property

$$
f(p)=\mathrm{NNDD}_{\text {RefSet }, \mathrm{d}, k, \theta, r}(p)
$$

holds. InRefSet and OutRefSet are sets used to partition the objects of the reference-consistent subset RefSet, as described in the following: Each object $p_{j}$ in OutRefSet has two associated heaps, $\delta_{j}$ and $\delta_{j}^{t}$, storing, respectively, the $k$ nearest neighbors of $p_{j}$ in RefSet and the $k$-nearest neighbors of $p_{j}$ in DataSet. Objects stored in InRefSet have no heaps associated.

First phase: first data set pass. During this step, the set OutRefSet stores the objects of RefSet such that $\left\|\delta_{j}\right\|_{r}=\left\|\delta_{\text {RefSet, }, k}\left(p_{j}\right)\right\|_{r}>\theta$, that is, the objects of the reference set that are rejected by the NNDD rule, whereas InRefSet contains the remaining objects of RefSet.

Furthermore, the heap $\delta$ associated with the current data set object $p_{i}$ stores the $k$-nearest neighbors of $p_{i}$ in RefSet. Hence, $\|\delta\|_{r}=\left\|\delta_{\text {RefSet }, \mathrm{d}, k}\left(p_{i}\right)\right\|_{r}$.

For each incoming data set object $p_{i}$, first of all, the distances among $p_{i}$ and the objects $p_{j}$ of OutRefSet are computed and the heaps $\delta_{j}^{t}$ are updated. Next, until the value $\|\delta\|_{r}$ remains above the threshold $\theta$, the distances among $p_{i}$ and the objects $p_{j}$ of InRefSet are computed. 
After having compared $p_{i}$ with the objects in OutRefSet and InRef Set, if $\|\delta\|_{r}$ remains above $\theta$, then $p_{i}$ is inserted in RefSet. ${ }^{2}$ In this case, the heap $\delta$ is updated with the object $p_{i}$, and the heaps $\delta_{i}$ and $\delta_{i}^{t}$ associated with $p_{i}$ are set equal to $\delta$. Furthermore, the heaps $\delta_{j}$ associated with the objects already contained in OutRefSet must be updated since now, $p_{i}$ belongs to RefSet: If after updating $\delta_{j}$, the value $\left\|\delta_{j}\right\|_{r}$ becomes less than or equal to $\theta$, then the object $p_{j}$ is removed from OutRefSet and inserted into InRef Set. In this case, the heaps $\delta_{j}$ and $\delta_{j}^{t}$ are no longer useful and can be discarded.

With RefSet $=$ OutRefSet $\cup$ InRefSet being a subset of DataSet, then it is the case that $\left\|\delta_{\text {DataSet, }, k}(p)\right\|_{r} \leq$ $\left\|\delta_{\text {RefSet,d, }, k}(p)\right\|_{r}$. Thus, the points $p$ of DataSet not stored in RefSet are such that $\left\|\delta_{\text {DataSet, } \mathrm{d}, k}(p)\right\|_{r} \leq\left\|\delta_{\text {RefSet, } \mathrm{d}, k}(p)\right\|_{r} \leq \theta$, and Property 1 is guaranteed for these objects. Therefore, at the end of the first scan, the objects of the data set not belonging to RefSet are correctly classified by it through the NNDD rule.

Furthermore, for each $p \in \overline{\mathcal{R}}(f), \theta<\left\|\delta_{\text {DataSet, }, k}(p)\right\|_{r} \leq$ $\left\|\delta_{\text {RefSet, }, k}(p)\right\|_{r}$, and, hence, RefSet (OutRefSet, to be more precise) contains the set $\overline{\mathcal{R}}(f)$.

Second phase: second data set pass. The first data set pass is not sufficient to assure consistency of the set RefSet since this set could contain objects that are misclassified by Ref Set itself. It could be the case that an object belonging to OutRefSet and, hence, rejected by the current RefSet is not an outlier.

Indeed, let $p_{j}$ be stored in OutRefSet at the end of the first scan. Unfortunately, $\left\|\delta_{j}^{t}\right\|_{r}>\theta$ does not imply that $p_{j} \in \overline{\mathcal{R}}(f)$, as $p_{j}$ was not compared with all the data set objects during the first data set pass. Thus, in order to establish whether $\left\|\delta_{\text {DataSet, }, k}\left(p_{j}\right)\right\|_{r}$ is greater than $\theta$, a second data set scan is performed.

For each $p_{j} \in$ OutRefSet, the heap $\delta_{j}^{t}$ is updated in order to compute the exact value of $\delta_{\text {DataSet,d,k}}\left(p_{j}\right)$ by comparing $p_{j}$ with all the objects $p_{i}$ in (DataSet - RefSet) such that $i<j$, that is, with the objects preceding $p_{j}$ that are not stored in RefSet. In fact, a generic object $p_{j}$ of OutRefSet was compared, during the first scan, exactly with all the objects $p_{i}$ of DataSet, where $j<i$ and with all the objects $\left\{p_{i} \in \operatorname{RefSet} \mid i<j\right\}$.

Third phase: reference set augmentation. The third phase of the algorithm is introduced to guarantee Property 1 for the objects stored in OutRefSet at the end of the first phase but determined to be inliers at the end of the second phase.

To this aim, the set RefSet is augmented with the set IncrRefSet until consistency is achieved. In particular, for each $p_{j} \in$ OutRefSet such that $\left\|\delta_{\text {DataSet, }, k}\left(p_{j}\right)\right\|_{r} \leq\left\|\delta_{j}^{t}\right\|_{r} \leq \theta$, that is, such that it is not an outlier, IncrRefSet is augmented with some nearest neighbors of $p_{j}$ until $\left\|\delta_{\text {RefSet } \cup \text { IncrRefSet, } \mathrm{d}, k}\left(p_{j}\right)\right\|_{r}$ goes down to the threshold $\theta$.

We note that, at the end of the algorithm, the objects in OutRefSet such that $\left\|\delta_{j}^{t}\right\|_{r}>\theta$ are the outliers of DataSet. This terminates the description of the CNNDD algorithm. The CNNDD rule is suitable for disk-resident data sets, as it tries to minimize the number of $\mathrm{I} / \mathrm{O}$ operations performing only two data set passes. As for the spatial cost of the CNNDD rule, it is $\mathcal{O}(\mid \operatorname{Re} f$ Set $\mid \cdot k)$, owing to the space needed to store heaps associated with objects in OutRefSet. The

2. Note that, as long as there are less than $k$ objects in the reference set Ref Set, the condition $\|\delta\|_{r}>\theta$ is always satisfied since at least one element of the vector $\delta$ is equal to $+\infty$, by definition of $k$-nearest neighbors distances vector. temporal cost is $\mathcal{O}(\mid$ RefSet $|\cdot|$ DataSet $\mid \cdot(d+\log k))$, where $d$ is the cost of computing the distance between two objects and $\log k$ is the cost of updating a heap of $k$ elements. The abovestated cost is the worst case, but usually, each data set object is compared only with a fraction of the objects in the reference subset. Thus, the temporal cost of the CNNDD rule depends on the size of the computed reference subset, and it is subquadratic in general, whereas it becomes quadratic when the reference subset consists of all the data set objects, that is, if we set $\theta=0$. As shown in the following section, for values of $\theta$ of interest, the reference-consistent subset is composed of a fraction of the data set objects.

\section{Experimental Results}

In this section, experiments involving the CNNDD rule are reported. Before starting, next, the data sets employed in the experiments are described. All the data sets are from the UCI Machine Learning Repository [10], ${ }^{3}$ except for the Checkerboard data set:

- Checkerboard. It is a synthetic data set composed of 2,000 randomly generated points of the unit square partitioned into two classes representing the cells of a $4 \times 4$ checkerboard.

- Image segmentation. This contains 2,310 objects representing $3 \times 3$ pixel regions obtained from a database of outdoor images. Each object has 19 continuous attributes. The objects are partitioned into seven classes, each composed of 330 objects: brickface, sky, foliage, cement, window, path, and grass.

- Ionosphere. This consists of radar data collected by a system in Goose Bay, Labrador. The 351 objects of this data set have 34 continuous attributes and are partitioned into two classes: good (225 objects associated with instances showing evidence of some type of structure in the ionosphere) and bad (126 objects).

- Iris. This data set contains three classes of 50 instances each. Each class refers to a type of iris plant: Setosa, Vericolour, and Virginica. The objects have four attributes representing the length and width of both sepal and petal.

- Letter recognition. The instances in this data set have 16 numerical attributes representing statistical moments and edge counts associated with black-andwhite images of the 26 capital letters of the English alphabet. The character images were based on 20 different fonts and each letter was randomly distorted to produce a total of 20,000 images. The objects in the data set are partitioned into 26 classes associated with the capital letters of the alphabet. Each class contains approximately the same number of instances.

- Satellite image. This consists of 6,435 instances (obtained by merging both the training and the test sets) generated from Landsat Multispectral Scanner image. Each object has 36 attributes consisting of multispectral values of pixels in $3 \times 3$ neighborhoods

3. Data sets were (somewhat randomly) selected among those whose objects are encoded as vectors of numeric attributes (since the CNNDD implementation that we had available manipulates only these kinds of object). 
in a satellite image. The class label is associated with the central pixel and may be one of the following: red soil (1,533 objects), cotton crop (703 objects), gray soil (1,358 objects), damp gray soil (626 objects), soil with vegetation stubble (707 objects), and very damp gray soil (1,508 objects).

- Shuttle. This data set was used in the European Statlog project. It contains eight attributes and 43, 500 instances. Approximately 80 percent of the data belongs to the class Rad Flow, which was assumed to represent the normal class, whereas the other instances belong to six different classes that were merged to obtain a single exceptional class.

- Vehicle. The features of this data set were extracted from the silhouettes of four types of vehicles. There are 18 attributes for each object. The data set consists of 846 object partitioned into the following four classes of vehicles: Opel (218 objects), Saab (217 objects), bus (199 objects), and van (212 objects).

- Wine. This data set is the result of a chemical analysis of wines grown in the same region in Italy but derived from three different cultivars (representing the three classes of the data set). The analysis determined the quantities of 13 constituents found in each of the three types of wines (the attributes of the objects). The objects are partitioned as follows: 59 objects into class 1,71 objects into class 2, and 48 objects into class 3.

The experiments are organized as follows: First of all, the behavior of the CNNDD is studied. Then, the CNNDD rule is compared with some one-class classification methods, namely, the NN-d method [25], the $k$-center method [28], and the one-class SVM algorithm [22]. Finally, the effect of Minkowski's metric $r$ used to compute the norm of the $k$-nearest neighbors distances vector and robustness to noise and outliers is investigated. In all the experiments, if not specified otherwise, the euclidean distance was used, whereas the parameter $r$ of the CNNDD method was set to 1 .

\subsection{The Effect of Condensing the Data Set}

In this section, the effect of condensing the reference set for the NNDD rule is investigated. Several experiments were performed. In each experiment, one of the data set classes described above was considered the normal one, whereas the other classes of the same data set formed the abnormal class.

During each experiment, the value of the parameter $k$ was varied between 1 and 10 and for each distinct value of $k$, the parameter $\theta$ was varied in the range $\left[0, \theta_{\max }\right]$. The value $\theta_{\max }$ depends on the data set considered.

For any combination of the parameters $k$ and $\theta$, the empirical error, the false-positive rate (f.p. for short in the following), and the detection rate (d.r. for short in the following) of both the NNDD and the CNNDD rules, and the size of the consistent reference subset, were computed.

The f.p. is the fraction of normal objects rejected by the classifier. The d.r. is the fraction of abnormal objects rejected by the classifier.

It must be recalled that, since it is assumed that the data set is composed only of normal objects, the abnormal objects are unknown at learning time. Both f.p. and d.r. were computed by 10 -fold cross validation.

Some of the experiments executed are reported in Fig. 2 (only one class for the data set since the behavior of the method on the other classes was analogous, as well as those for $k=1$ and $k=5$ in order to show the effect of increasing the value of the parameter $k$ ). The $x$-axis reports the threshold value $\theta$, whereas the $y$-axis varies between 0 and 1 and reports the f.p., the d.r., the empirical error, and the normalized size of the reference-consistent subsets.

Solid and dotted lines represent the f.p. and the d.r. of the CNNDD rule, respectively. Dashed and dash-dotted lines represent the f.p. and the d.r. of the NNDD rule, respectively. The empirical error is represented by the dashed pointed lines. Finally, the solid pointed lines report the normalized size of the reference-consistent subset computed by the CNNDD rule.

From these figures, it is clear that, for relatively large values of parameter $\theta$, the CNNDD rule noticeably improves the d.r. over the NNDD rule with a little loss, or even with no loss, of f.p. Furthermore, in the same range of values of $\theta$, the training set compression achieved by using the CNNDD rule is noticeable. Depending on the data set of interest (and on the desired trade-off between d.r. and f.p.), the size of the reference-consistent subset ranges from a few percent of the overall training set up to 10-20 percent of the training set objects. It can thus be concluded that the consistent reference subset guarantees remarkable reference set size reduction. The best trade-off between classifier accuracy and compression ratio is achieved in the curve elbow of the f.p.

Furthermore, as expected, when $\theta$ approaches 0 , both the f.p. and the d.r. approach 1 , whereas the consistent reference subset computed by the CNNDD rule tends to contain all the data set objects, as they are almost all outliers.

By observing Fig. 2, it can be concluded that, when a certain value of the f.p. is fixed by using a value for parameter $k$, which is greater than 1 , may improve the d.r. achievable at the expense of an increase in the size of the reference-consistent subset.

Interestingly, it can be noticed that, for example, in the Image segmentation path data set, for $k=5$ and $\theta=2$, the CNNDD method achieves a d.r. of 0.984 , whereas the d.r. of the NNDD method was about 0.907 with practically no loss of the f.p. (0.015 of CNNDD versus 0.012 of NNDD) and a reference set that is less than 10 percent of the whole data set. As a further example, consider the Wine class 1 data set for $k=5$ and $\theta=4$, where the CNNDD improves the d.r. from 0.575 of the NNDD rule to about 0.948 by employing a subset of only 11 percent of the data set. Also, consider the Letter recognition data set for $\theta \in[20,40]$ and $k=5$. The same behavior can be observed in other experiments shown and also in the experiments concerning the data set classes not reported here owing to space limitations.

If both normal and abnormal data are provided, then a suitable combination of values for the parameters can be determined by executing the same kind of experiment described above. Nevertheless, with the method being unsupervised, it can be employed when only normal data is available. In this case, as holds for any other unsupervised method, it can be difficult to determine the accurate value for the parameters.

However, in the case of the CNNDD rule, the following procedure can be profitably applied. As far as the value of parameter $\theta$ is concerned, the value $\theta^{*}$ can be used such that the slope of the curve of the threshold $\theta$ versus the normalized size of the reference-consistent subset $\rho=$ $|S| /|D|$ is equal to a user-provided value $\alpha$. As far as the 

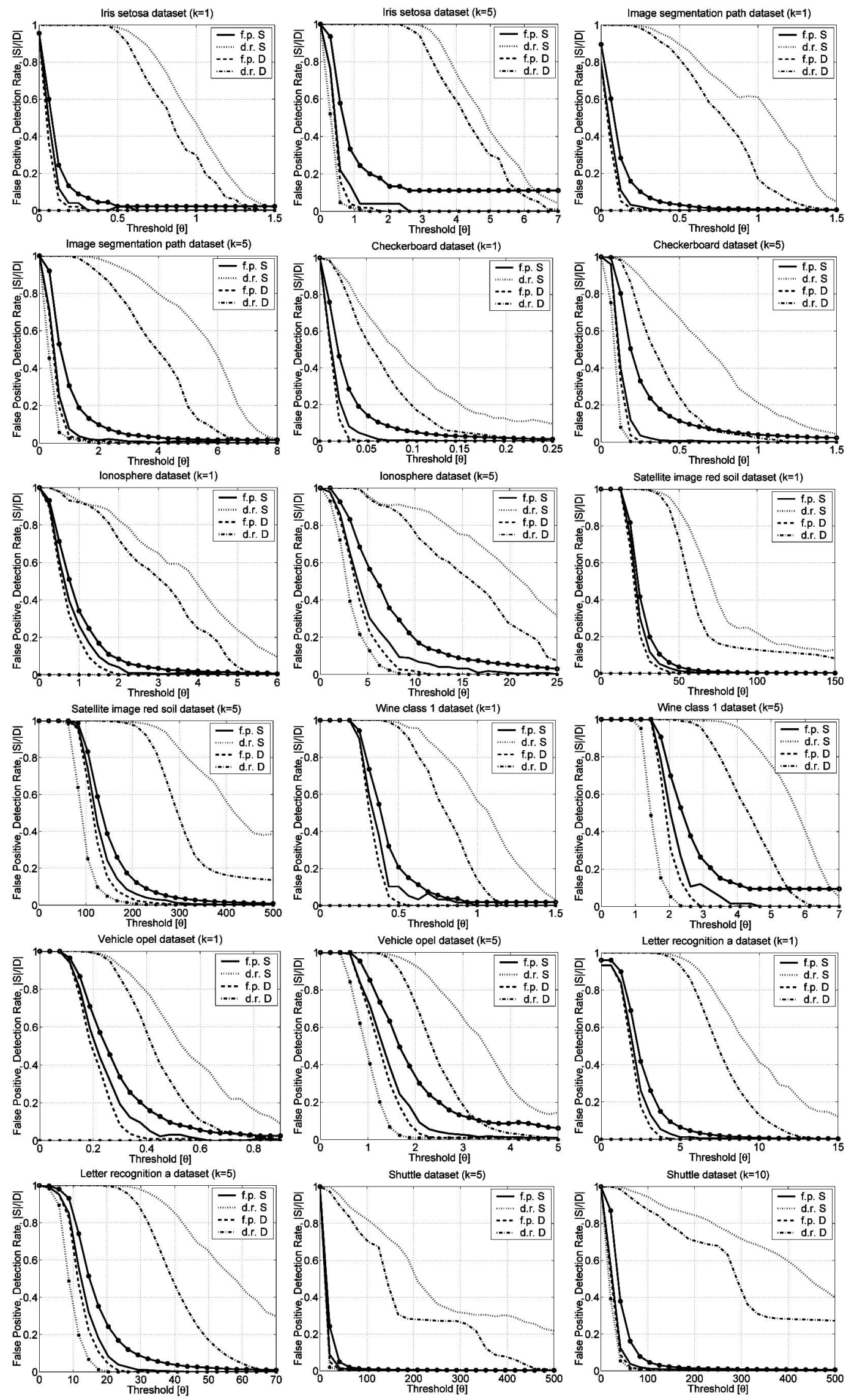

Fig. 2. Comparison between the CNNDD and the NNDD rules. 


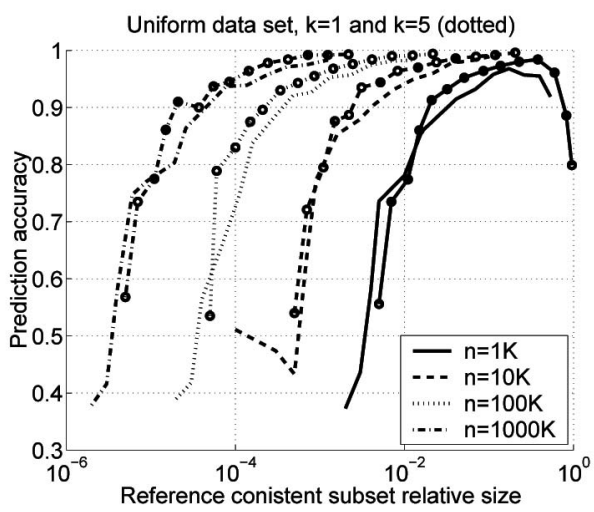

Fig. 3. How the compression ratio and the prediction accuracy vary with the training set size on a uniform data set.

value of parameter $k$ is concerned, the value $k^{*} \geq 1$ can be used such that the size of the reference-consistent subset achieved for $\theta=\theta^{*}$ does not exceed a user-provided ratio $\rho_{\max }$. As can be verified on the curves in Fig. 2, using $\alpha \approx$ $45^{\circ}$ and $\rho_{\max } \leq 0.2$ provides a good classifier in almost all the experiments.

Before concluding this section, it is studied how the condensation ratio and the prediction accuracy achieved by the CNNDD method vary, together with the training set size. With this aim, a family of synthetically generated training sets, called Uniform data set in the following, was considered. Each training set of the family is composed of $n$ points $(n \in\{1 K, 10 K, 100 K, 1,000 K\})$ uniformly distributed into the square $[0.25,0.75]^{2}$ in which it is assumed to represent the normal class. To measure the prediction accuracy, 1,000 uniformly distributed random points of the unit square were employed. For each training set and $k \in\{1,5\}$, the parameter $\theta$ was varied to obtain different consistent subsets, and the prediction accuracy achieved was measured. Fig. 3 shows the curves of the referenceconsistent subset relative size versus the prediction accuracy. It is worth noticing that the condensation ratio increases dramatically with increasing training set size, whereas the quality of the prediction remains unchanged, and that by increasing the parameter $k$ (the dotted lines are those for $k=5$ ), the accuracy of the classifier is improved.

Fig. 4 shows some examples of training-set-consistent subsets computed by the CNNDD algorithm on the Uniform data set composed of $n=1,000 K$ points, together with the misclassified points of the test set. For clarity, the training set points are not shown in the figure. The dashed curve represents the square $[0.25,0.75]^{2}$ partitioning the points of the unit square into two classes. The small crosses represent the consistent subset points, whereas the dots are the test points. Circles are the test points misclassified by the consistent subset. From left to right and from top to bottom, the sizes of the consistent subset are 23, 78, 792, 16, 144, and 795, whereas the prediction accuracies are 0.844, 0.937, $0.977,0.876,0.964$, and 0.991 , respectively. It is clear that, by decreasing the value of $\theta$, the number of points composing the consistent subset increases, whereas the prediction accuracy is improved.

\subsection{Comparison with Other Approaches}

In this section, the CNNDD rule is compared with the NN-d [25], the $k$-center [28], and the one-class SVM [22] classifiers. These methods are described first.

The one-class classifier NN-d accepts a test object when its local density is greater than or equal to the local density of its $k$ th nearest neighbor. The local density of an object is estimated by computing the distance between the object
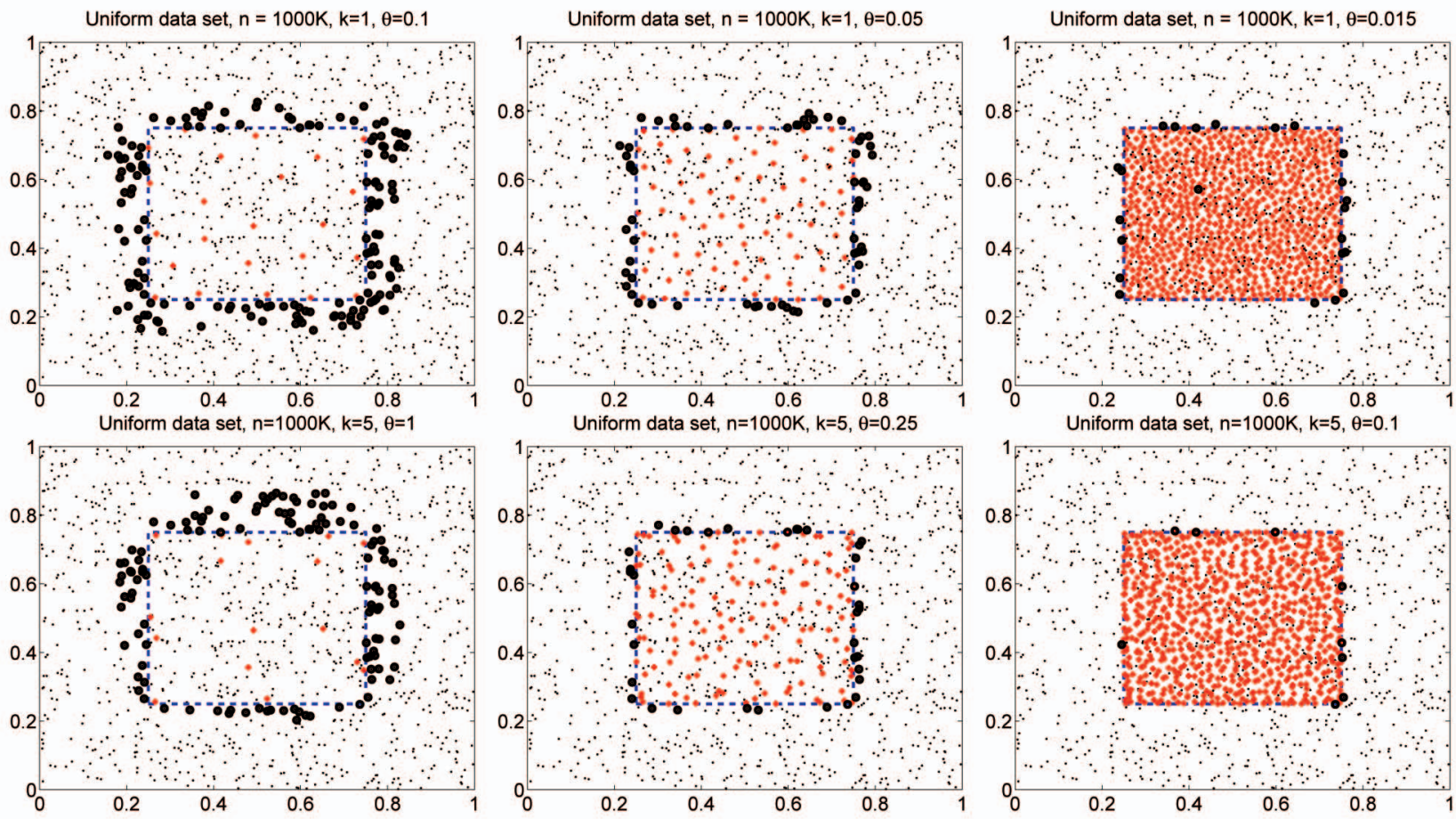

Fig. 4. Training-set-consistent subsets of the Uniform data set for various values of the parameters and points misclassified during prediction. 
TABLE 1

Comparison of the CNNDD, the NN-d, and the One-Class SVM Methods through ROC Areas

\begin{tabular}{|l|c|c|c|c|}
\hline Data set & CNNDD & NN-d & $k$-center & one-class SVM \\
\hline \hline Checkerboard & $0.978(k=7)$ & $0.937(k=4)$ & 0.917 & $0.946(\gamma=50.0)$ \\
\hline \hline Image segmentation brickface & $0.998(k=1)$ & $0.983(k=10)$ & 0.994 & $0.870(\gamma=2.00)$ \\
Image segmentation sky & $1.000(k=2)$ & $0.999(k=3)$ & 0.998 & $0.995(\gamma=0.20)$ \\
Image segmentation foliage & $0.962(k=1)$ & $0.928(k=3)$ & 0.845 & $0.891(\gamma=5.00)$ \\
Image segmentation cement & $0.961(k=3)$ & $0.876(k=2)$ & 0.855 & $0.907(\gamma=2.00)$ \\
Image segmentation window & $0.958(k=2)$ & $0.926(k=2)$ & 0.859 & $0.890(\gamma=5.00)$ \\
Image segmentation path & $0.999(k=2)$ & $0.995(k=3)$ & 0.997 & $0.982(\gamma=1.00)$ \\
Image segmentation grass & $0.997(k=2)$ & $0.995(k=6)$ & 0.995 & $0.956(\gamma=0.001)$ \\
\hline \hline Ionosphere good & $0.959(k=10)$ & $0.914(k=9)$ & 0.937 & $0.918(\gamma=0.10)$ \\
\hline \hline Iris setosa & $1.000(k=1)$ & $0.989(k=3)$ & 0.980 & $0.947(\gamma=0.01)$ \\
Iris versicolor & $0.981(k=5)$ & $0.958(k=7)$ & 0.963 & $0.980(\gamma=0.20)$ \\
Iris virginica & $0.960(k=5)$ & $0.926(k=4)$ & 0.921 & $0.937(\gamma=0.10)$ \\
\hline \hline Letter recognition A & $0.998(k=3)$ & $0.980(k=4)$ & 0.992 & \\
\hline \hline Satellite image red soil & $0.993(k=1)$ & $0.938(k=5)$ & 0.983 & $0.974(\gamma=0.0001)$ \\
Satellite image cotton crop & $0.985(k=5)$ & $0.849(k=2)$ & 0.965 & $0.940(\gamma=0.0001)$ \\
Satellite image grey soil & $0.974(k=5)$ & $0.936(k=3)$ & 0.953 & $0.963(\gamma=0.0002)$ \\
Satellite image damp grey soil & $0.891(k=5)$ & $0.819(k=1)$ & 0.806 & $0.911(\gamma=0.0001)$ \\
Satellite image soil with vegetation stubble & $0.944(k=5)$ & $0.900(k=1)$ & 0.848 & $0.851(\gamma=0.0002)$ \\
Satellite image very damp grey soil & $0.950(k=4)$ & $0.874(k=2)$ & 0.889 & $0.942(\gamma=0.0001)$ \\
\hline \hline Shuttle & $0.995(k=2)$ & $0.995(k=3)$ & - & \\
\hline \hline Vehicle opel & $0.961(k=5)$ & $0.937(k=3)$ & 0.919 & $0.868(\gamma=2.00)$ \\
Vehicle saab & $0.751(k=5)$ & $0.698(k=4)$ & 0.647 & $0.686(\gamma=5.00)$ \\
Vehicle bus & $0.935(k=2)$ & $0.912(k=3)$ & 0.909 & $0.835(\gamma=3.00)$ \\
Vehicle van & $0.740(k=2)$ & $0.692(k=1)$ & 0.719 & $0.649(\gamma=5.00)$ \\
\hline \hline Wine class 1 & $0.998(k=5)$ & $0.993(k=7)$ & 0.964 & $0.949(\gamma=0.20)$ \\
Wine class 2 & $0.916(k=5)$ & $0.844(k=4)$ & 0.857 & $0.847(\gamma=0.10)$ \\
Wine class 3 & $0.994(k=3)$ & $0.988(k=1)$ & 0.975 & $0.958(\gamma=0.50)$ \\
\hline
\end{tabular}

and its $k$ th nearest neighbor in the reference set without $q$. Thus, given a test object $p$, the NN-d method accepts $p$ if

$$
\frac{\mathrm{d}\left(p, n n_{k}(p, D)\right)}{\mathrm{d}\left(n n_{k}(p, D), n n_{k+1}\left(n n_{k}(p, D), D\right)\right)} \leq \theta
$$

and rejects it otherwise $(k=1$ and $\theta=1$ are usually employed).

The $k$-center one-class method covers the data set with $k$ balls having equal radii. Ball centers $\mu_{j}$ are placed on training objects such that the maximum distance of all minimum distances between the training objects and the centers is minimized, whereas all the objects are covered by some balls; that is, the following measure is minimized:

$$
\rho=\max _{p_{i} \in D}\left[\min _{j=1}^{k} \mathrm{~d}\left(p_{i}, \mu_{j}\right)\right] .
$$

The radius $\rho$ defines the boundary of the target class around the $k$ selected centers.

The one-class SVM algorithm is a specialization, working in the presence of only positive data, of the standard two-class SVM algorithm that, conversely, requires both positive and negative examples. Basically, the feature space is transformed via a kernel, and then, the origin of the transformed space is treated as the only member of the negative class. Thereafter, the standard two-class SVM algorithm is employed. The oneclass SVM exploits the parameter $\nu \in(0,1]$ in order to control the trade-off between the number of training set examples accepted and the size of the SV regularization term. Specifically, parameter $\nu$ is both an upper bound on the fraction of outliers and a lower bound on the fraction of SVs. The LibSVM [5] implementation of the one-class SVM was used in the experiments presented.
Before starting the comparison, it is of interest to point out the major differences between $k$-center and CNNDD since, among the methods described above, $k$-center is the most similar to the method here introduced. In particular, three aspects are to be taken into account: The form of the decision boundary, the treatment of outliers and the algorithmic approaches.

First, the form of the decision boundary of the $k$-center and the CNNDD methods is different. Indeed, the accepting region of the $k$-center is the union of $k$ balls centered at some data set objects. Conversely, the accepting region of the CNNDD is the union of at most $\left(\begin{array}{l}n \\ k\end{array}\right)$ regions, each associated with $k$ distinct objects of the condensed set. For example, in a normed linear space, if the norm $r=1$ is employed, then it can be shown that these regions are convex sets "generated" by $k$ elements of the condensed set. Thus, for $k=2$ and $r=1$, the accepting region of the CNNDD is the union of hyperellipses. For $k>2$, more complex regions, describing a boundary around the $k$ points, are generated. As a consequence, the decision boundary of the CNNDD may be more accurate than that of $k$-center, and this is especially evident whenever the objects of the target class are close to the objects of the other classes, and/or the boundary is particularly complex, and also in the presence of noise or outliers (see Table 1). For example, let an ellipse on the plane represent the accepting region of the target class. This region can be described exactly by the CNNDD with a subset composed of two objects (the focus of the ellipse), whereas the number of balls to be used by the $k$-center must approach infinity to reach the same level of detail.

Second, the $k$-center always accepts all the training set objects, whereas the CNNDD classifier rejects a fraction of the training set objects, directly proportional to the value of $k$ and 

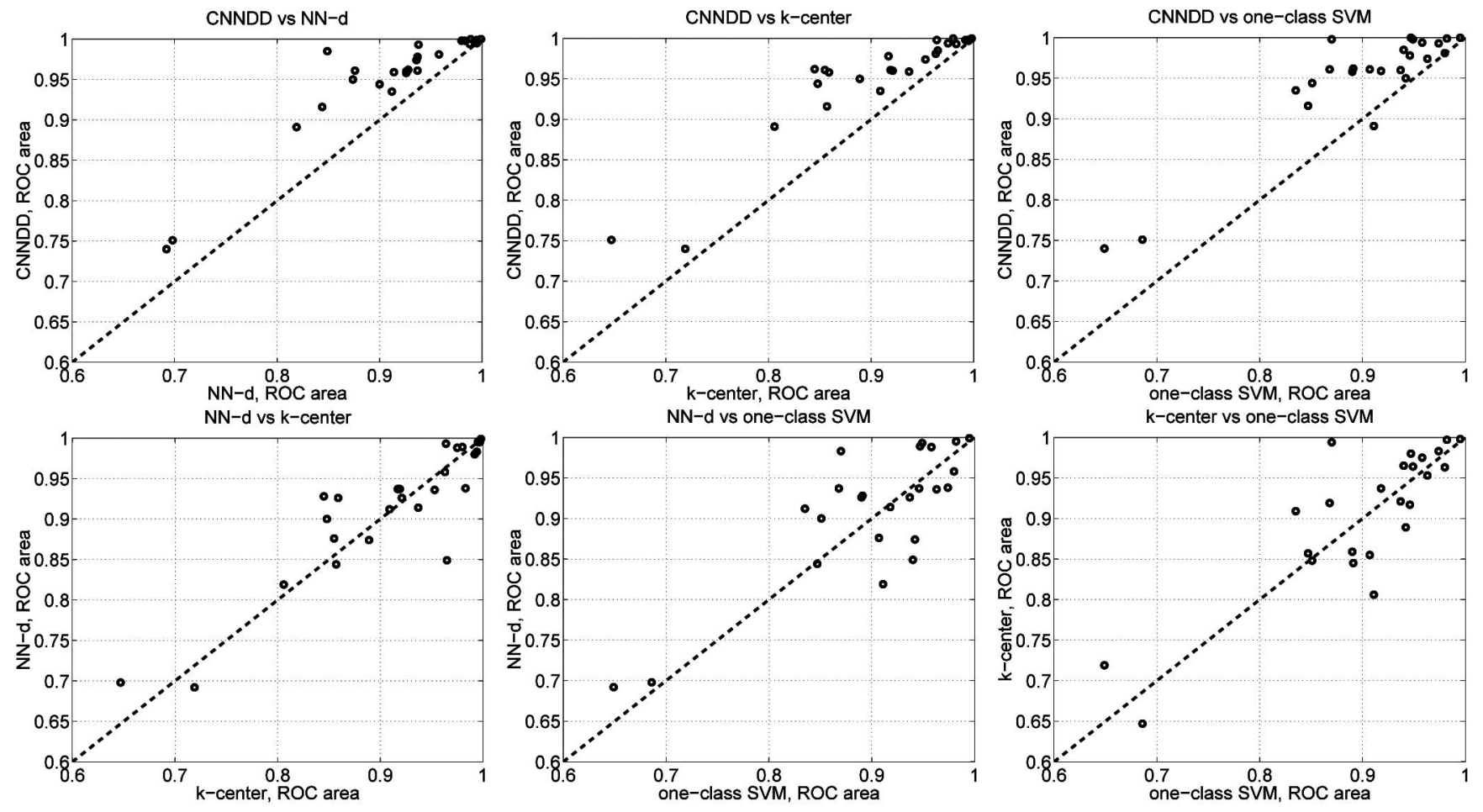

Fig. 5. Pairwise comparison of the ROC areas of the CNNDD, the NN-d, $k$-center, and the one-class SVM methods.

inversely proportional to the value of $\theta$. This implies that the $k$-center is more sensitive to noise than the CNNDD. As an example, consider a training set composed of 1,000 objects very close to each other, thus forming a cluster, plus a single outlying object far from the cluster. Although the CNNDD method, with $k=2$ and a value of $\theta$ smaller than the distance separating the cluster from the outlier, covers the cluster with a hyperelliptical region and leaves the outlier out of its accepting region, the $k$-center method, with $k=2$, covers the objects with two spheres: The first is centered at the cluster and the second is centered at the outlier. With the neighborhood of the outlier being included in the accepting region of the $k$-center classifier, it is clear that it may degrade prediction accuracy, especially if it falls into the support of a different class. Furthermore, it follows from what is stated above that the CNNDD can be used to detect outliers in the input data set, whereas it is not the case in the $k$-center.

Finally, even by disregarding these basic differences between the two methods, it is clear that, since the $k$-center fixes the number $k$ of centers in advance, whereas the CNNDD fixes the threshold $\theta$ used to define the boundary around the subset objects, different algorithmic approaches are feasible to solve the two problems efficiently.

The comparison of the methods was made through the receiver operating characteristic (ROC) analysis. ROC curves are the plot of the f.p. versus the d.r. and the area under the curve gives an estimate of the ability of the method to separate inliers from outliers. ROC curves are computed by 10 -fold cross validation.

As far as the CNNDD and the NN-d methods are concerned, parameter $k$ was varied between 1 and 10, and for each value of $k$, parameter $\theta$ was varied between 0 and $\theta_{\max }$. As for the $k$-center method, the number $k$ of centers was varied between 1 and the 90 percent of the training set objects.
Regarding the one-class SVM, parameter $\nu$ was varied between a value close to 0 and 1.0, where $n$ denotes the number of training set objects, and the radial basis function (RBF) kernel was used, varying parameter $\gamma$ between $10^{-4}$ and $10^{2}$. For each method, the best area under the ROC curves computed, as described above, was then determined.

Table 1 shows the ROC areas of the three methods (together with the values for the parameters used to achieve that area). Interestingly, in all the data sets considered, the CNNDD rule performed better than the other three methods.

As far as the one-class SVM method is concerned, in various experiments, it was observed that, although for relatively small values of parameter $\gamma$, the ROC curve is not as good as the curve of the CNNDD method, for relatively large values of $\gamma$, they are comparable. Nevertheless, often, the curve of the one-class SVM method is not defined for small values of f.p. (up to 5-10 percent) and, hence, it is less accurate, and eventually, it scores a smaller ROC area.

As far as the NN-d method is concerned, its curve is always below the curve of the CNNDD method and as a result, it scores a smaller ROC area. Furthermore, it must be pointed out that the NN-d rule uses all the data set objects as reference set.

A similar behavior was observed also for the $k$-center method. Basic differences between the $k$-center and the CNNDD were previously discussed.

Table 1 does not show the ROC areas of the one-class SVM on the Letter recognition A and Shuttle data sets and of the $k$-center on the Shuttle data set, since computing them required too much computational effort.

The results presented in Table 1 are summarized in Fig. 5, where a pairwise comparison of the four methods is accomplished. Each point $(x, y)$ represents a distinct experiment such that $x$ is the ROC area of the method on the 

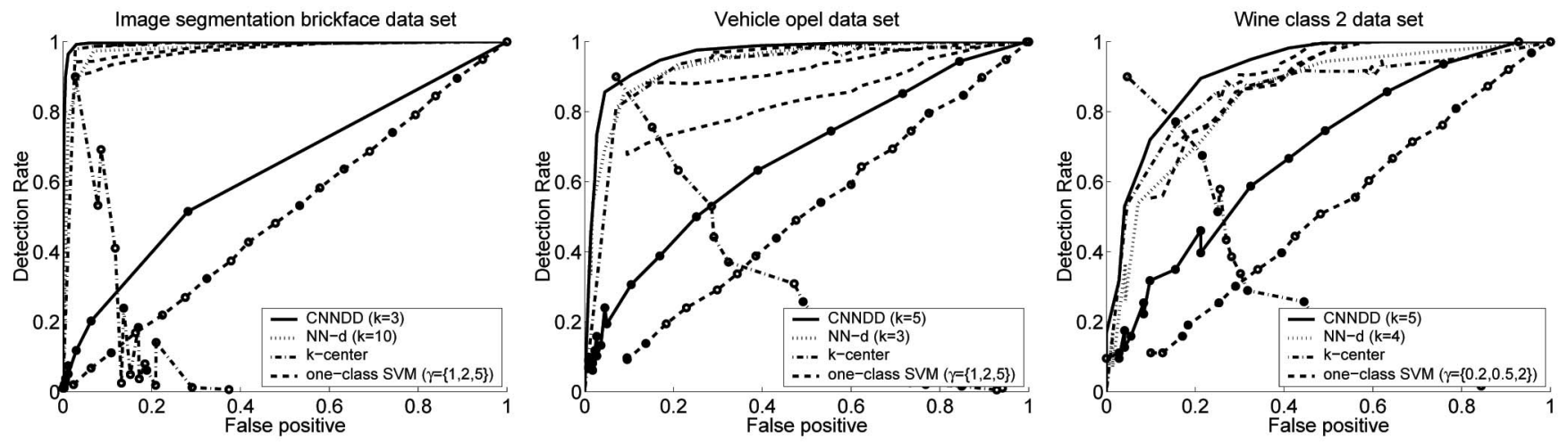

Fig. 6. Comparison of the CNNDD, the NN-d, $k$-center, and the one-class SVM methods: ROC curves and percentage of objects composing the model.

abscissa, and $y$ is the ROC area of the method on the ordinate. If the two methods are comparable, then the points will be symmetrically distributed along the dashed line connecting $(0,0)$ to $(1,1)$ and partitioning the unit square in two regions. Otherwise, the points will lie mostly in one of the two abovedefined regions. It is clear from these diagrams that the CNNDD rule outperformed the other three methods in the experiments considered, whereas the $\mathrm{NN}-\mathrm{d}$, the $k$-center, and the one-class SVM methods appear to be comparable on these experiments.

Fig. 6 compares the ROC curves of the CNNDD (solid line), the NN-d (dotted line), the $k$-center (dash-dotted line), and the one-class SVM (dashed lines) algorithms and the compression ratios achieved by the CNNDD (solid pointed line), the $k$-center (dash-dotted pointed line), and the oneclass SVM (dashed pointed line) methods.

These curves confirm the behavior described above. Indeed, the ROC curves of the NN-d and the $k$-center methods were always below the ROC curve of the CNNDD method. As far as the one-class SVM is concerned, it is needed to increase the value of parameter $\gamma$ of the RBF kernel in order to achieve a curve comparable to that of the CNNDD method. However, as parameter $\gamma$ increased, we were not able to
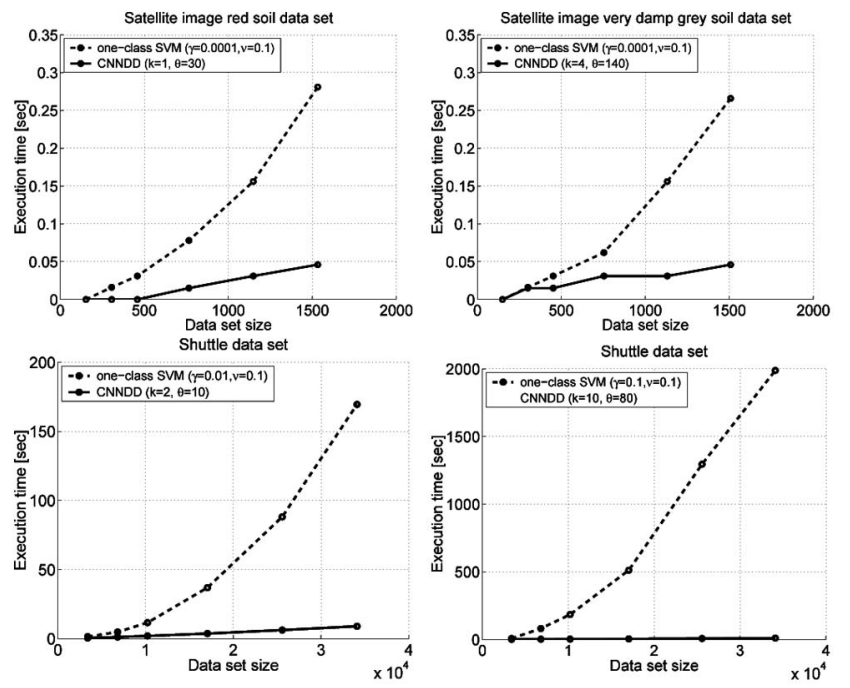

Fig. 7. Comparison of the execution times of the CNNDD and of the oneclass SVM. obtain a curve defined for all the values of the f.p. because the curve became undefined up to 5-10 percent of the f.p. This is an undesirable behavior since usually this is just the range of values for the f.p. that one is interested in achieving after parameter tuning as a result of the training phase.

As far as the compression ratios are concerned, as expected, the percentage of SVs of the one-class SVM is approximately equal to the f.p. It can be noticed that the number of objects composing the CNNDD reference subset was always greater than the number of SVs. We were not able to realize whether this behavior is an intrinsic property of the minimum-sized NNDD reference-consistent subset or otherwise if it is due to the fact that the CNNDD rule is a greedy method computing an approximate solution, that is, a reference-consistent subset, which is, in general, not minimum (recall that the problem of computing the minimum one is intractable). In any case, for low values of the f.p., the size of the reference-consistent subset returned by the CNNDD method is slightly greater than the number of SVs. Furthermore, it must be noticed that the lower compression ratio of the CNNDD is repaid by an ROC curve, which is much more accurate. As for the compression ratio of the $k$-center method, it is clear that, in order to obtain a good f.p./d.r. trade-off, the number $k$ of data objects to be employed as centers of the classifier is high, that is, at least 80 percent, as in the experiments reported in Fig. 6.

Finally, Fig. 7 shows the scaling behavior of the CNNDD algorithm and of the learning phase of the one-class SVM. ${ }^{4}$ Execution times are those reported by the LibSVM implementation of the one-class SVM. We considered the Satellite image and Shuttle data sets since they are the largest data sets among those employed in the experiments.

As for the one-class SVM, the curves reported are obtained by fixing the parameter $\nu$ to 0.1 . As for the CNNDD method, the value of $\theta$ is such so that the CNNDD rule scores 0.1 f.p.

As for the Satellite image data set, the value of $\gamma$ is that associated with the best d.r. achieved by the one-class SVM, whereas the value of $k$ is that associated with the best d.r. achieved by the CNNDD rule.

As for the Shuttle data set, the curve for $k=2$ of the CNNDD method (those associated with its best ROC area)

4. Experiments were performed on a Pentium Mobile 1700-MHz-based machine having 1 Gbyte of main memory and running on the Windows XP operating system. 


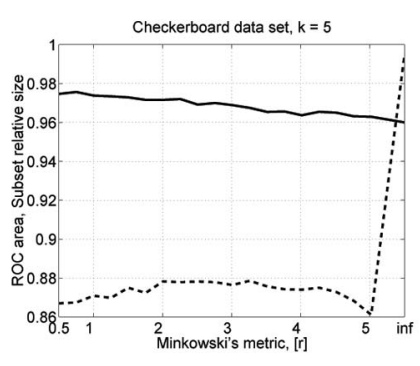

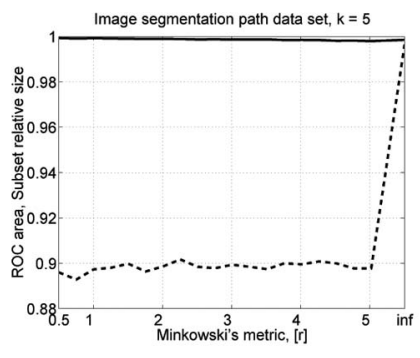

(a)

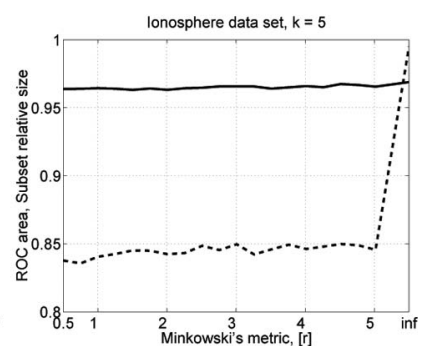

lonosphere data set, $k=5$

(b)

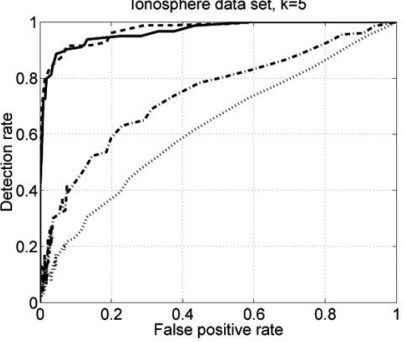

Fig. 8. Sensitivity to the Minkowski's metric $r$.

is compared with the curve for $\gamma=0.01$ of the one-class SVM and also the curve for $k=10$ of the former method with the curve for $\gamma=0.1$ of the latter method.

It can be observed that, although the CNNDD algorithm seems to scale nearly linearly, training the one-class SVM is costly. Indeed, on the whole data set, the latter method can be one or two orders of magnitude slower than the former, whereas, owing to the different trend of the two curves, the ratio between the execution times of the two methods is even expected to increase if larger data sets are to be considered.

For example, on the Shuttle data set composed of 34,108 objects having nine features each, the one-class SVM, with parameters $\nu=0.1$ and $\gamma=0.01$, required about 170 seconds versus the 9 seconds of the CNNDD rule, with parameters $k=2$ and $\theta=10$ (f.p. $=0.1$ ). Furthermore, on the same data set, the one-class SVM for $\nu=0.1$ and $\gamma=0.1$ required about 2,000 seconds versus the 10 seconds of the CNNDD rule for $k=10$ and $\theta=80$ (f.p. $=0.1$ ).

\subsection{Sensitivity to the Norm and Robustness to Noise}

In this section, two important aspects of the CNNDD method are investigated, that is, the effect of Minkowski's metric $r$ used to compute the norm of the $k$-nearest neighbors distances vector and the robustness of the method to noise or outliers possibly belonging to the reference set.

First of all, the effect of norm $r$ is considered. In order to measure the sensitivity of the CNNDD to this parameter, $r$ was varied in the range $[0.5,5] \cup\{+\infty\}$ and both the area under the ROC curve and the area under the curve of the false positive ratio versus the condensation ratio, say, $\tau$ area, were measured. Results concerning the Checkerboard, Ionosphere, and Image segmentation path data sets are shown in Fig. $8(k=5$ was used in all the experiments). The solid curve represents the area under the ROC curve, whereas the dashed curve represents the ratio $\tau / \tau_{\max }$, where $\tau_{\max }$ denotes the greatest value of $\tau$ encountered.

As for the accuracy, although for the Ionosphere and for the Image segmentation path, it remained practically unchanged, for the Checkerboard data set, by augmenting the value of $r$, the accuracy gradually and slightly worsened. Thus, the accuracy of the CNNDD appears to have low sensitivity to the choice of norm $r$. However, it must be noticed that for small values of $r(r \leq 1)$, the accepting region of CNNDD follows the distribution of the reference set points more closely than for greater values of $r$. Since the two classes composing the Checkerboard data set are very close, and the boundary is quite complex (it is the union of some axis-parallel segments), this explains why for low values of $r$ the method performed slightly well. The same behavior has not been observed in the other experiments since the classes are better separated.

As for the condensation ratio $\tau$, for all the data sets, it remained almost constant for $r<+\infty\left(\tau / \tau_{\max }\right.$ is approximately 0.85-0.9), whereas for $r=+\infty$, it reached its maximum value $\tau_{\max }$. Thus, it can be concluded that by using the infinity metrics, the condensation ratio worsens. This behavior can be explained by noticing that, from the data reduction point of view, this norm is more demanding than the other norms. Indeed, under the $r=+\infty$ metrics, an object can be discarded from the reference set only if at least $k$ objects lie within distance $\theta$ from it, whereas for any other metrics $r$, the same object can be discarded, provided that there exist $k$ objects such that the sum $\sum_{i}\left(d_{i}\right)^{r}$ of their distances $d_{i}$ from it is less than $\theta^{r}$. It is evident that the former is a more severe condition than the latter. The value $\tau / \tau_{\max }$ is a short summary of the condensation achieved, but it does not represent a real ratio between sizes of reference-consistent subsets (recall that it is an area). In order to visualize this ratio, Fig. $8 \mathrm{~b}$ reports for $r=1$ and $r=+\infty$, the ROC curves (solid $r=1$, dashed $r=+\infty$ ) and the curves of $\tau$ (dotted: $r=1$, dash-dotted: $r=+\infty$ ) obtained on the Ionosphere data set. Although the ROC curves are very close, depending on the level of the f.p., the ratio between the sizes of the consistent subsets may change noticeably. For example, for values of the f.p. around 0.1, the size of the subset doubles if the infinity metrics is employed. A similar behavior was observed also on the other data sets. It can be concluded that the value $r=1$, used in the rest of the paper, is, in general, a good choice for this parameter.

In order to study the robustness of the CNNDD method to noise, two experiments were executed.

First of all, the Uniform data set (10,000 2D points) previously described was considered as a training set. An inlier and an outlier test sets were generated to measure the f.p. and the d.r. The inlier data set is composed of 10,000 2D points into the square $A=[0.25,0.75]^{2}$. The outlier data set is composed of 10,000 points into the region $B=[0,1]^{2}-A$. Then, noisy versions of the Uniform training set were obtained by adding from 1 percent (100 points) to 10 percent (1,000 points) of whitelike noise (randomly generated points belonging to $B$ ). For values of $k$ ranging from 1 to 5 , the ROC curves associated with the training sets with noise were computed. Fig. 9a reports on these curves. On the top left, there are curves for $k=1$ and on the top center are curves for $k=5$. It is clear that by adding noise, the accuracy of the CNNDD decreases though even by adding considerable amount of noise, the rule produces a good classifier. Importantly, as the figure on the top right shows (relative to 10 percent of noise), by increasing the value of $k$, the effect of the noise is mitigated, and the resulting classifier is of remarkable quality. 

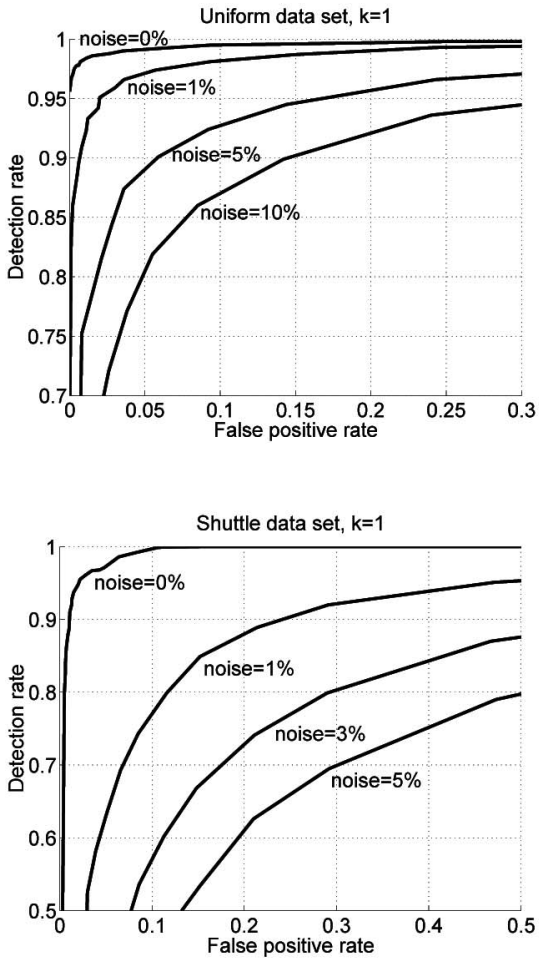

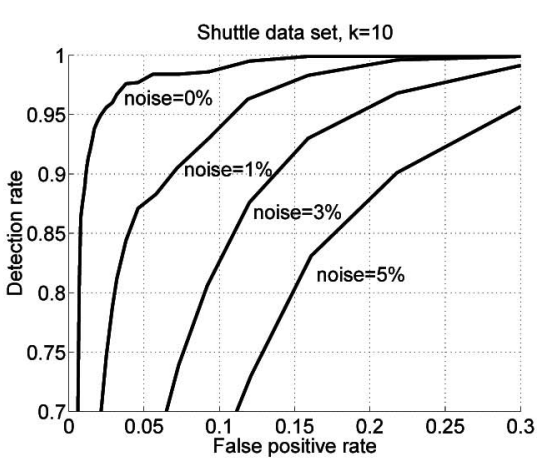

(b)

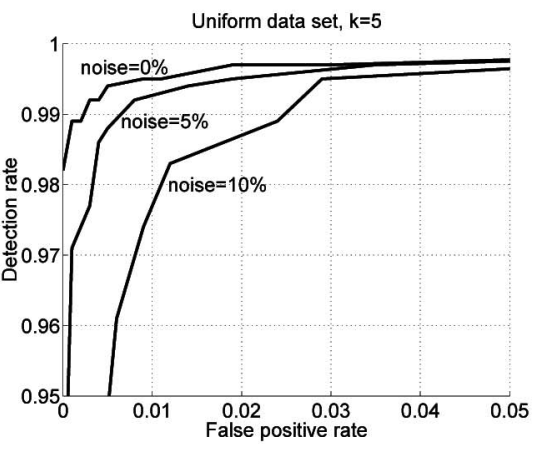

(a)
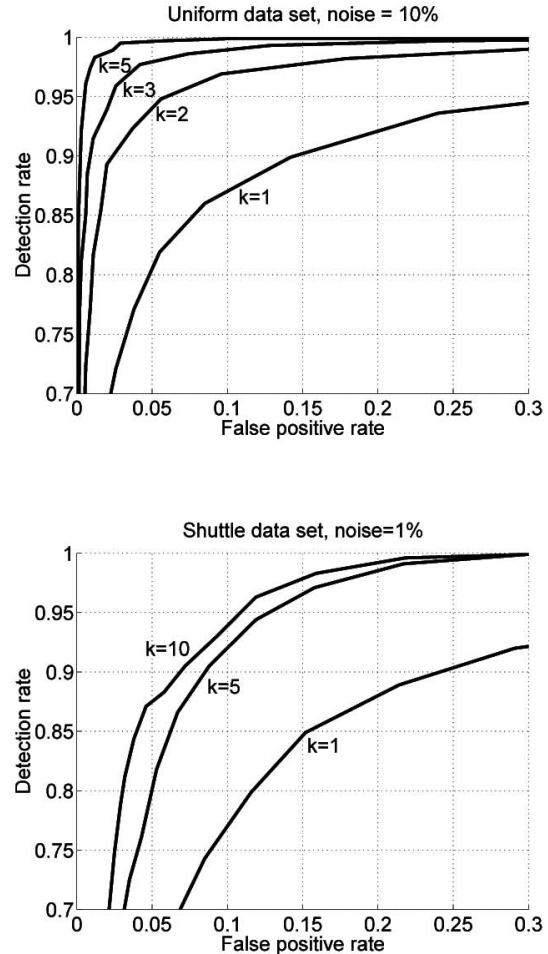

Fig. 9. Robustness to noise.

An analogous experiment was executed on the Shuttle data set. The 34,108 points of the Rad Flow class were equally partitioned in a training set and in an inlier test set (of 17,054 points each), whereas the 9,392 points of the other classes and in an outlier test set were formed. Then, noisy versions of the training set were obtained by adding from 1 percent (170 points) to 5 percent ( 850 points) of mislabeled points (randomly selected points belonging to the outlier test set). Also, in this case, for values of $k$ ranging from 1 to 10 , the ROC curve associated with the training sets with outliers were computed (reported in Fig. 9b). On the bottom left, there are curves for $k=1$, on the bottom center are curves for $k=10$, whereas on the bottom right are curves relative to 1 percent of noise. By observing these curves, it is clear that the behavior of the method is unchanged even if "biased" noise is added. Furthermore, recall that in this second experiment, the noisy points added are points belonging to the outlier test set, rather than points coming from the whole feature space.

It can be concluded that increasing the parameter $k$ has the positive outcome of mitigating the impact of possibly noise and outliers, thus noticeably improving classifier accuracy. Indeed, as previously noted, by increasing $k$, the objects lying in the less densely populated regions of the feature space are rejected and no longer contribute to form the accepting region of the classifier.

\section{Conclusions}

In this paper, the behavior of one-class classification based on a nearest neighbor training-set-consistent subset has been investigated. With this aim, the concept of the referenceconsistent subset has been introduced, and the computational complexity of its computation has been investigated. A fast greedy algorithm named CNNDD was described, which computes a reference-consistent subset with only two reference set passes. Comprehensive experimental activity revealed strengths and weaknesses of the method.

As a future work, several extensions of the research done here are worthy of being examined, such as methods for computing subsets of a size close to the minimum one, investigating the different definitions of subsets, which may improve generalization, applying the technique of condensation to other nearest-neighbor-based one-class classification methods, and exploring the power of feature selection in conjunction with training set condensation.

\section{ACKNOWLEDGMENTS}

The author would like to thank the anonymous reviewers for their comments that greatly helped in improving the presentation of the work.

\section{References}

[1] F. Angiulli, "Fast Condensed Nearest Neighbor Rule," Proc. 22nd Int'l Conf. Machine Learning, pp. 7-11, Aug. 2005.

[2] F. Angiulli and C. Pizzuti, "Fast Outlier Detection in HighDimensional Spaces," Proc. European Conf. Principles and Practice of Knowledge Discovery in Databases, pp. 15-26, 2002.

[3] M. Breunig, H.P. Kriegel, R. Ng, and J. Sander, "Lof: Identifying Density-Based Local Outliers," Proc. ACM Int'l Conf. Management of Data, 2000.

[4] V. Cerverón and F.J. Ferri, "Another Move toward the Minimum Consistent Subset: A Tabu Search Approach to the Condensed Nearest Neighbor Rule," IEEE Trans. Systems, Man, and Cybernetics —Part B: Cybernetics, vol. 31, no. 3, pp. 304-408, 2001.

[5] C.-C. Chang and C.-J. Lin, "LIBSVM: A Library for Support Vector Machines," 2001, http://www.csie.ntu.edu.tw/ cjlin/libsvm. 
[6] T.M. Cover and P.E. Hart, “Nearest Neighbor Pattern Classification," IEEE Trans. Information Theory, vol. 13, pp. 21-27, 1967.

[7] B. Dasarathy, "Minimal Consistent Subset (MCS) Identification for Optimal Nearest Neighbor Decision Systems Design," IEEE Trans. Systems, Man, and Cybernetics, vol. 24, no. 3, pp. 511-517, 1994.

[8] L. Devroye, "On the Inequality of Cover and Hart," IEEE Trans. Pattern Analysis and Machine Intelligence, vol. 3, pp. 75-78, 1981.

[9] L. Devroye, L. Gyorfi, and G. Lugosi, A Probabilistic Theory of Pattern Recognition. Springer-Verlag, 1996.

[10] C.L. Blake, D.J. Newman, S. Hettich, and C.J. Merz, "UCI Repository of Machine Learning Databases," 1998.

[11] E. Eskin, A. Arnold, M. Prerau, L. Portnoy, and S. Stolfo, "A Geometric Framework for Unsupervised Anomaly Detection: Detecting Intrusions in Unlabeled Data," Applications of Data Mining in Computer Security, 2002.

[12] E. Fix and J. Hodges, "Discriminatory Analysis," Non-Parametric Discrimination: Consistency Properties, Technical Report 4, School of Aviation Medicine, US Air Force (USAF), Randolph Field, Texas, 1951.

[13] S. Floyd and M. Warmuth, "Sample Compression, Learnability and the Vapnik-Chervonenkis Dimension," Machine Learning, vol. 21, no. 3, pp. 269-304, 1995.

[14] M.R. Garey and D.S. Johnson, Computer and Intractability. W.H. Freeman and Co., 1979.

[15] P.E. Hart, "The Condensed Nearest Neighbor Rule," IEEE Trans. Information Theory, vol. 14, pp. 515-516, 1968.

[16] D.S. Hochbaum and D.B. Shmoys, "A Best Possible Heuristic for the $k$-Center Problem," Math. Operations Research, vol. 10, no. 2, pp. 180-184, 1985.

[17] E. Knorr and R. Ng, "Algorithms for Mining Distance-Based Outliers in Large Datasets," Proc. Int'l Conf. Very Large Databases, pp. 392-403, 1998.

[18] N. Littlestone and M. Warmuth, "Relating Data Compression and Learnability," technical report, Univ. of California, Santa Cruz, 1986.

[19] S. Ramaswamy, R. Rastogi, and K. Shim, "Efficient Algorithms for Mining Outliers from Large Data Sets," Proc. ACM Int'l Conf. Management of Data, pp. 427-438, 2000.

[20] G. Ritter and M.T. Gallegos, "Outliers in Statistical Pattern Recognition and an Application to Automatic Chromosome Classification," Pattern Recognition Letters, vol. 18, pp. 525-539, Apr. 1997.
[21] B. Schölkopf, C. Burges, and V. Vapnik, "Extracting Support Data for a Given Task," Proc. Int'l Conf. Knowledge Discovery and Data Mining, pp. 251-256, 1995.

[22] B. Schölkopf, J. Platt, J. Shawe-Taylor, A.J. Smola, and R.C. Williamson, "Estimating the Support of a High-Dimensional Distribution," Technical Report 87, Microsoft Research, Redmond, Wash., 1999.

[23] C. Stone, "Consistent Nonparametric Regression," Annals of Statistics, vol. 8, pp. 1348-1360, 1977.

[24] D. Tax and R. Duin, "Data Domain Description Using Support Vectors," Proc. European Symp. Artificial Neural Networks, pp. 251256, Apr. 1999.

[25] D. Tax and R. Duin, "Data Descriptions in Subspaces," Proc. Int'l Conf. Pattern Recognition, pp. 672-675, 2000.

[26] D.M.J. Tax, "One-Class Classification," PhD dissertation, Delft Univ. of Technology, June 2001.

[27] G. Toussaint, "Proximity Graphs for Nearest Neighbor Decision Rules: Recent Progress," Technical Report SOCS-02.5, School of Computer Science, McGill Univ., Montreal, Québec, 2002.

[28] A. Ypma and R. Duin, "Support Objects for Domain Approximation," Proc. Internet Corporation for Assigned Names and NumbersConf., 1998.

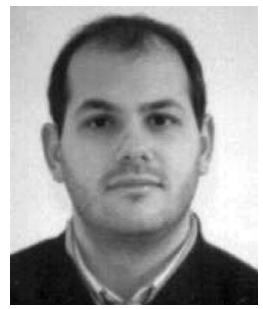

Fabrizio Angiulli received the Laurea degree in computer engineering from the University of Calabria, Italy, in 1999. From January 2001 to August 2006, he was with the Institute of HighPerformance Computing and Networking of the Italian National Research Council (ICAR-CNR). From September 2006, he worked as an assistant professor in the Dipartimento di Elettronica Informatica e Sistemistica (DEIS), University of Calabria. He is also a lecturer at the University of Catanzaro. His research interests include artificial intelligence, data mining, machine learning, and databases.

$\triangleright$ For more information on this or any other computing topic, please visit our Digital Library at www.computer.org/publications/dlib. 